\title{
Quantification and a Simple Correction of Perspective Error in Two-Dimensional Motion Analysis of Human Movement
}

\author{
TAKASHI YOKOI ${ }^{1}$ AND HIDETAKA OKADA ${ }^{2}$ \\ 1 National Institute of Bioscience and Human Technology, 1-1 Higashi, \\ Tsukuba, Ibaraki 305, Japan \\ 2 Institute of Health and Sport Sciences, University of Tsukuba, 1-1-1 Ten- \\ oudai, Tsukuba, Ibaraki 305, Japan
}

Received October 22, 1993

\begin{abstract}
The perspective error in two-dimensional motion analysis based on film and/or video images was theoretically quantified, and the practical effects of the error on the accuracy in two-dimensional kinematic variables were investigated using the data of sprint running. The equations proposed in the present study may be useful for estimating the amount of perspective error and determining appropriate camera distance in two-dimensional motion analysis. In practical analysis of human movement, the error affects translational and rotational kinematics of limb segments. If the optical axis of the camera is perpendicular to the calibration plane and the intersection of the axis and plane can be detected, the effects of perspective error on translational kinematics for the limb segments can be reduced to a certain extent.
\end{abstract}

Key Words: perspective error, two-dimensional measurement, human movement, kinematic analysis

\section{INTRODUCTION}

The motion analysis of human movement using video images as well as cinematography are indispensable for the quantitative description of various human movements and to identify the characteristics of the movement. Although threedimensional motion analysis recently has become popular in biomechanical research, two-dimensional analysis based on the images obtained through a single camera is still used in anthropological and biomechanical studies because the cost, time and equipment necessary for both experiment and analysis are less.

In the filming for two-dimensional quantitative motion analysis, human movement is projected into a principal photographic plane, which is used for scale calibration. The real human movement and/or the human body, however, is three dimensional. Accordingly, it may be necessary to consider perspective error for obtaining accurate real coordinates from two dimensionally filmed images. Perspective error occurs when parts of the body or implements lie outside the principal plane. As a result, the image of the arm or leg closer to the camera will be larger 
than that of the corresponding limbs on the opposite side of the body even though they have the same size in real space. This type of error is also present when a limb is at an angle to the photographic plane and hence appears shorter than when it is situated exactly within the calibration plane.

Many articles exist on the perspective error in motion analysis. The error may have effects on various kinematic variables such as calibrated two-dimensional coordinates of body landmarks and joint angles (Plagenhoef, 1966; Noss, 1967; Miller and Nelson, 1973). The perspective errors contained in the values are larger for the digitized coordinates located near the boundary of the filmed area. The distance from the camera to the objects as well as the object's depth along the optical axis of the camera also have effects on the perspective errors. Since the perspective error is not a random error, it cannot be eliminated through data smoothing procedures. Noss (1967) made qualitative consideration of angle data affected by perspective error and recommended the use of three-dimensional measurement techniques for eliminating the effect on angles. For reducing the perspective error in two-dimensional data acquisition, Miller and Nelson (1973) as well as Plagenhoef (1968) recommended positioning the camera as far away from the filmed objects as possible. Unfortunately, few studies have exactly quantified the effects of perspective error on kinematic variables concerning human movement. Although placing the camera far from the subject may be practical for outdoor experiments, it is not appropriate for indoor experiments in which camera distance is limited by the space of experiment room. It is obvious that perspective errors exist in indoor experiments. It is not clear, however, that considerations for perspective error were made in recent two-dimensional studies on human locomotion (Iwata, 1983; Yamada et al., 1984).

Recently, automatic marker tracking systems have become available for both twodimensional and three-dimensional motion measurements. These systems detect the markers, which contrast with the background by light reflection, light sources or light absorption. For the appropriate use of such a system, it may be necessary to regulate the lighting condition. Thus, the system needs to be used indoors, and the problem on perspective error becomes inevitable in any two-dimensional motion measurement using the automatic marker tracking system.

Considering the above situations, it seems necessary to quantify the perspective error and reconfirm the existence of the error for appropriate data analysis and subsequently, reliable conclusions. Also, it is necessary, from a practical viewpoint, to identify the effects of the perspective error on kinematic variables.

The purpose of the present study was to quantify the perspective errors contained in the estimated two-dimensional coordinates of landmark locations. We also examined the effects of perspective errors on the accuracy of two-dimensional kinematic variables, and provided a simple and practical method to eliminate the perspective error in quantitative two-dimensional motion measurement. 


\section{METHODS}

\section{Theoretical considerations}

In two-dimensional motion analysis, the scale calibration is usually performed using a proportional calibration method based on the scale factor (Miller and Nelson, 1973) and/or the direct linear transformation method (Walton, 1979). In the following theoretical consideration, we mainly used the proportional method. As a result, the optical axis of camera is assumed to be perpendicular to the calibration plane. The two-dimensional direct linear transformation method is thought to be a special case of the calibration using proportional method as far as the optical axis is perpendicular to the calibration plane. Thus, the results obtained in the present study can also be applied to the perspective error in a two-dimensional direct linear transformation method following the calibration condition above described. We also assume that the errors due to lens distortion and random errors resulting from the digitizing process are negligible.

We consider the perspective error under the filming situation shown in Fig. 1. The camera is located at a distance $\mathrm{D}$ from the calibration plane. Object 0 is located within the calibration plane. Object 1 is farther from the camera while object 2 is closer. Objects 1 and 2 are D+d and D-d from the camera, respectively, where $d$ is the distance of these objects from the calibration plane. The apparent image size for objects 0,1 and 2, which have the same true size $R$, are assumed to be $r_{0}, r_{1}$ and $r_{2}$, respectively.

The image size $r_{0}, r_{1}$ and $r_{2}$ on the film plane can be expressed by using $L_{f}, R$, $\mathrm{D}$ and $\mathrm{d}$ :

$$
\begin{aligned}
& \mathrm{r}_{0}=\mathrm{L}_{\mathrm{f}} \cdot \mathrm{R} / \mathrm{D} \\
& \mathrm{r}_{1}=\mathrm{L}_{\mathrm{f}} \cdot \mathrm{R} /(\mathrm{D}+\mathrm{d}) \\
& \mathrm{r}_{2}=\mathrm{L}_{\mathrm{f}} \cdot \mathrm{R} /(\mathrm{D}-\mathrm{d}),
\end{aligned}
$$

where $\mathrm{L}_{\mathrm{f}}$ is the focal length of camera.

In order to calculate the real object size through the proportional method, we usually use a scale factor c expressed by equation (2).

$$
\mathrm{c}=\mathrm{R} / \mathrm{r}_{0}
$$

The estimated true size for objects 0,1 and 2 are given:

$$
\begin{aligned}
& \mathrm{R}_{0}=\mathrm{c} \cdot \mathrm{r}_{0}=\mathrm{R} \\
& \mathrm{R}_{1}=\mathrm{c} \cdot \mathrm{r}_{1}=\mathrm{D} \cdot \mathrm{R} /(\mathrm{D}+\mathrm{d}) \\
& \mathrm{R}_{2}=\mathrm{c} \cdot \mathrm{r}_{2}=\mathrm{D} \cdot \mathrm{R} /(\mathrm{D}-\mathrm{d})
\end{aligned}
$$

If the objects are in the calibration plane, the estimated size is equal to $R$ as shown in equation ( $3 a$ ). However, the estimated sizes $R_{1}$ and $R_{2}$ are not equal to $R$ since objects 1 and 2 are outside the calibration plane. In this case, the relative perspective 


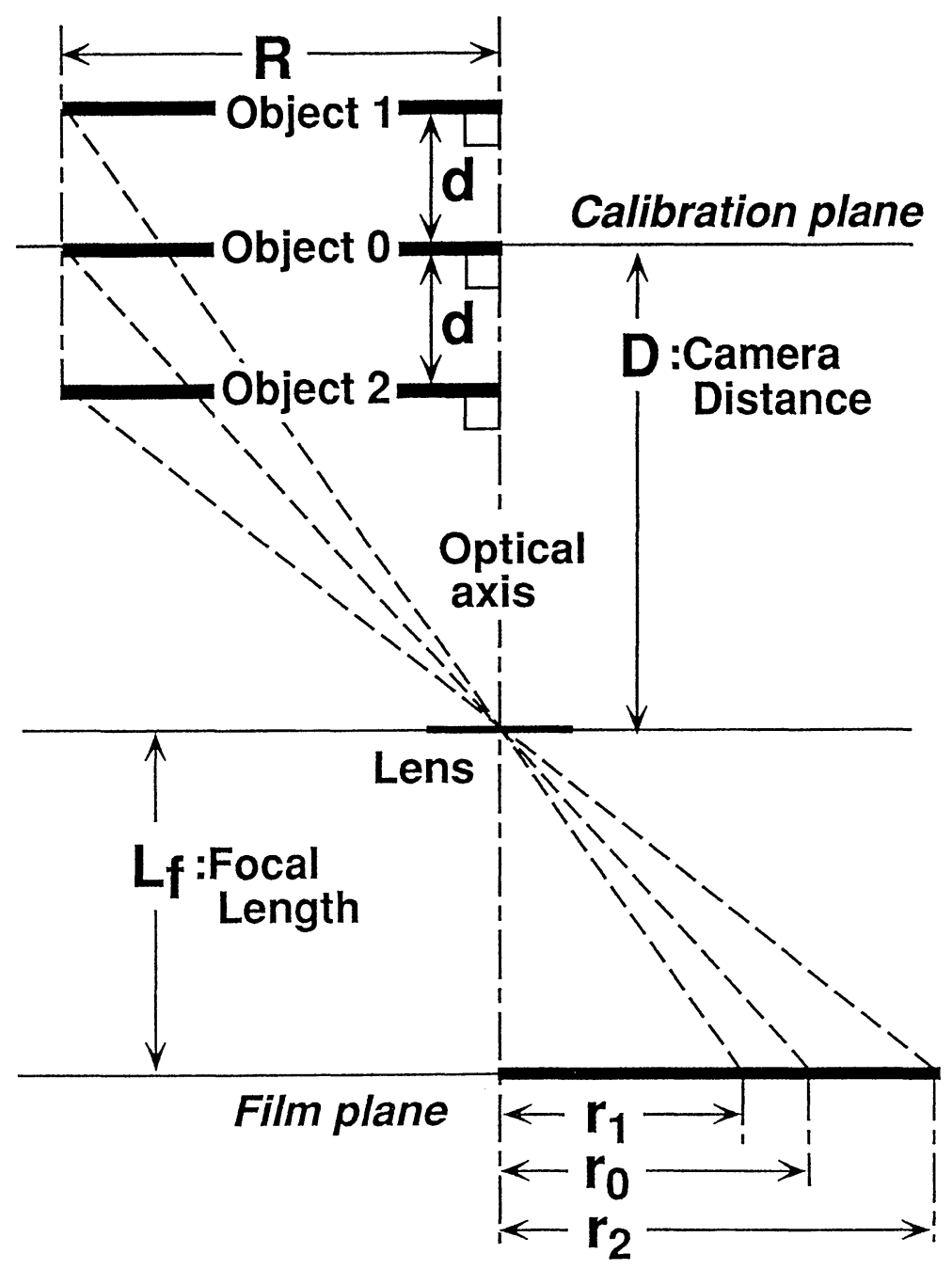

Fig. 1. The filming situation considered in the present study and the relationship between three objects of the same sizes and their apparent images.

error for objects 1 and 2 are

$$
\begin{array}{ll}
\text { Object 1: } & e_{1}=100 \cdot\left(R_{1}-R_{0}\right) / R_{0}=-100 \cdot d /(D+d) \\
\text { Object 2: } & e_{2}=100 \cdot\left(R_{2}-R_{0}\right) / R_{0}=100 \cdot d /(D-d)=E .
\end{array}
$$

These errors are expressed by a percentage of real object size. The values of $e_{1}$ and $\mathrm{e}_{2}$ depend only on the camera distance D and the object's depth d. Obviously, 
$\left|e_{1}\right| \leq\left|e_{2}\right|$, and the relative perspective errors does not exceed $\pm e_{2}$ as far as the objects are located within the range $\mathrm{D} \pm d$ from the camera. Thus, we adopted $e_{2}$ as a parameter to evaluate perspective errors in the filming for two-dimensional motion analysis, and accordingly, we refer to $\mathrm{e}_{2}$ as $\mathrm{E}$ in the following sections.

Solving the equation (4b) for D,

$$
\mathrm{D}=(100+\mathrm{E}) \cdot \mathrm{d} / \mathrm{E} \text {. }
$$

This equation can be used to determine the appropriate camera distance $\mathrm{D}$ in order to diminish the perspective error within $E$ when filming objects with depth $d$.

\section{Simulation of a two-dimensional kinematic analysis}

In order to examine the practical effects of perspective errors on kinematic variables, three dimensional coordinates of body landmarks during several steps of sprint running were used as a reference data set. The sprint running was filmed at $60.6 \mathrm{~Hz}$ with two $16 \mathrm{~mm}$ high-speed cinecameras. The three-dimensional coordinates of body landmarks were estimated by using the direct linear transformation method (Walton, 1979), and the coordinates data were digitally smoothed at $7.0 \mathrm{~Hz}$.

Horizontal anteroposterior and vertical components of the three-dimensional coordinates were used as real two-dimensional coordinates. For the simulation of the two-dimensional coordinates containing perspective errors, the calibration plane was assumed to be a vertical plane including three dimensional locus of the whole body center of gravity during the sprinting. The camera is supposed to be located at the distances $D=5 \mathrm{~m}, 10 \mathrm{~m}, 20 \mathrm{~m}, 40 \mathrm{~m}$ and $80 \mathrm{~m}$ from the calibration plane, which is perpendicular to the optical axis of the camera. The origins for both spatial and planar coordinate systems were assumed to be the intersection of the optical axis and the calibration plane.

Figure 2 shows the relationship between the three-dimensional coordinates (X, $\mathrm{D} \pm \mathrm{d}, \mathrm{Z}$ ) and the simulated two-dimensional coordinates (u, v) which contain the perspective errors. $\mathrm{D}$ indicates the camera distance from the calibration plane and $\mathrm{d}$ is the distance between a body landmark and the calibration plane. $\mathrm{X}$ is the horizontal anteroposterior component, and $\mathrm{Z}$ is the vertical component of the coordinates. The coordinates $(\mathrm{u}, \mathrm{v})$ can be calculated from $(\mathrm{X}, \mathrm{D} \pm \mathrm{d}, \mathrm{Z})$ based on the following equations;

$$
\begin{aligned}
& \mathrm{u}=\mathrm{D} \cdot \mathrm{X} /(\mathrm{D} \pm \mathrm{d}) \\
& \mathrm{v}=\mathrm{D} \cdot \mathrm{Z} /(\mathrm{D} \pm \mathrm{d}),
\end{aligned}
$$

while the real two-dimensional coordinates are $(\mathrm{X}, \mathrm{Z})$. The simulated twodimensional coordinates $\left(\mathrm{u}_{\mathrm{i}}, \mathrm{v}_{\mathrm{i}}\right)$ of a body landmark $\mathrm{i}$ were computed frame by frame for each camera distance.

Several kinematic variables such as joint angles were computed using both 


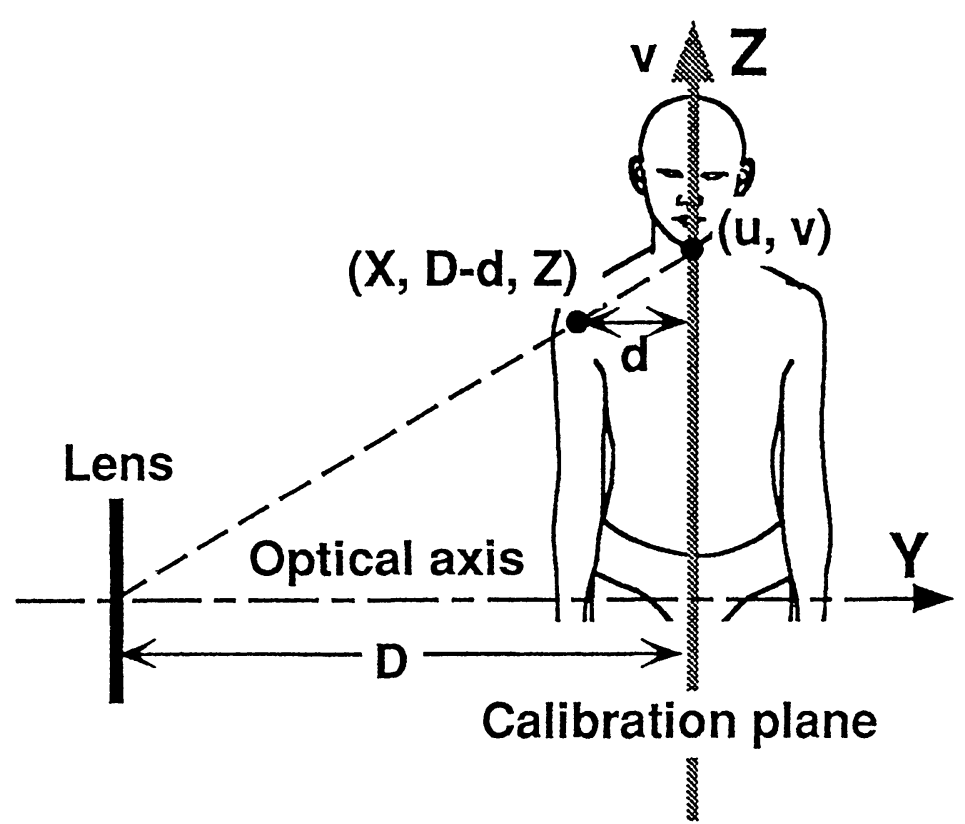

Fig. 2. Relation between the real three-dimensional coordinates (X, D-d, Z) of a body landmark and the two-dimensional coordinates $(u, v)$. The coordinates $(u, v)$ include perspective error.

$\left(\mathrm{u}_{\mathrm{i}}, \mathrm{v}_{\mathrm{i}}\right)$ and $\left(\mathrm{X}_{\mathrm{i}}, \mathrm{Z}_{\mathrm{i}}\right)$. Then, we discussed the practical effects of the perspective error on the kinematic variables through a comparison of the variables in the simulated and real two-dimensional data set under the assumption that the real two-dimensional data include no error.

\section{RESULTS AND DISCUSSION}

\section{Effects of camera location and object size on perspective error}

The perspective error, $E$ defined by equation (4b), increased with camera distance (D), as shown in Fig. 3a. The error is illustrated for three depths: $d= \pm 0.1 \mathrm{~m}$, $\pm 0.3 \mathrm{~m}, \pm 0.5 \mathrm{~m}$. Obviously, the perspective error is inversely proportional to $\mathrm{D}$. The error also decreased as $\mathrm{d}$ become smaller. Figure $3 \mathrm{~b}$ shows the predicted relation between $\mathrm{d}$ and $\mathrm{D}$ (equation (4c)) for perspective errors of $1 \%, 3 \%$ and $5 \%$. The relationship in Fig. $3 \mathrm{~b}$ indicates, for example, that in order to reduce perspective error to $1 \%$, the camera distance must be greater than $40 \mathrm{~m}$ when filming objects with $0.4 \mathrm{~m}$ depth.

Figure $3 \mathrm{a}$ and equation (4b) can be used for the estimating perspective error in a practical filming situations. On the other hand, Fig. $3 b$ and equation (4c) may be 
a

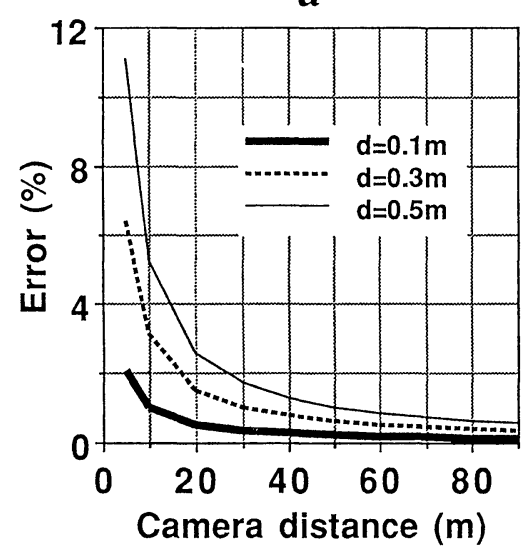

b

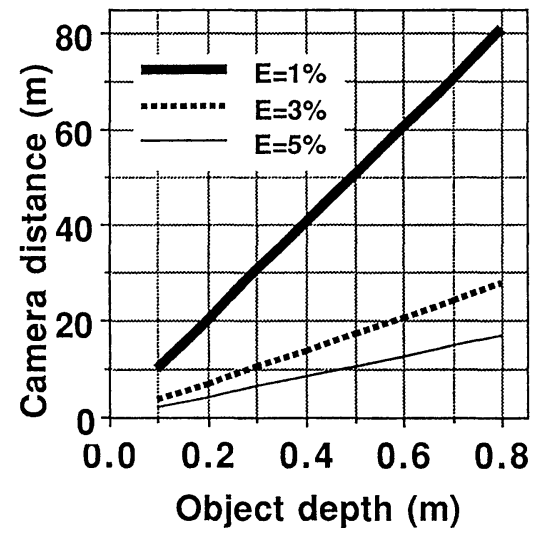

Fig. 3. Relation among perspective error, camera distance and object depth. a: relationship between perspective error and camera distance for $0.1,0.3$ and $0.5 \mathrm{~m}$ of object depth (d). b: relationship between camera distance and object depth for 1,3 and $5 \%$ of perspective error (E).

useful for determining the camera distance appropriate for filming objects having a certain depth. In the two-dimensional analysis of walking and running, the filmed movement is usually supposed to take place in the median sagittal plane of the subject. Assuming the depth of subject and/or motion is around $\pm 0.4 \mathrm{~m}$, the perspective error (E) is about $5 \%$ of movement range if the filming is done at a camera distance of $10 \mathrm{~m}$. This indicates that the coordinates of landmarks within $2 \mathrm{~m} \times 2 \mathrm{~m}$ of the filmed area (in the X-Z plane) inevitably include around $0.1 \mathrm{~m}$ of perspective error in filming at distances of $10 \mathrm{~m}$. If the perspective error included in the coordinates of body landmarks is required to be less than $1 \%$ of the filmed area, the camera distance must be more than $40 \mathrm{~m}$.

We also tried to estimate the amount of perspective error which occurs due to the experimental setup and analysis procedures in the two-dimensional locomotion study reported by Iwata (1983). In that study, the camera distance was around $9 \mathrm{~m}$, and the locomotion movement was executed about $1 \mathrm{~m}$ in front of the calibration plane, i.e., $8 \mathrm{~m}$ from the camera. Thus, substituting the values, $\mathrm{D}=9 \mathrm{~m}$ and $\mathrm{d}=1 \mathrm{~m}$, into the equation (4b), we obtained a perspective error of $12.5 \%$. If Iwata did not correct the perspective error, this value indicates that the displacements and velocities of the locomotion would be $112.5 \%$ of the true values. Considering the experimental setup of the study, the error can be reduced through the correction of the scaling factor used in her study. The $12 \%$ deviation cannot be regarded as small. Iwata, however, gave no account of any correction of the scaling factor. 


\section{Effects of perspective error on the kinematic variables for sprint running}

Although the amount of perspective error contained in the two-dimensional coordinates was quantitatively estimated in the previous consideration, the practical effects of the error on kinematic variables in human movement are not yet clear. In the following, we consider the effects on several kinematic variables in sprinting motion.

The deviations in step length (SL) and running speed (RS) with the increase of camera distance are shown in Fig. 4. SL was obtained as half of the horizontal progress of the right toe during one cycle, defined as right foot contact, and RS was computed as the product of SL and step frequency. Although the camera distance is increased from $5 \mathrm{~m}$ to $80 \mathrm{~m}$, neither SL or RS changed much. Actually, the discrepancy between real and simulated data in the investigated camera conditions was less than $0.02 \mathrm{~m}$ for SL and $0.08 \mathrm{~m} / \mathrm{s}$ for RS. These small discrepancies may have occurred due to the fact that the toe motion in sprinting occurs almost in the median sagittal plane of the subject.

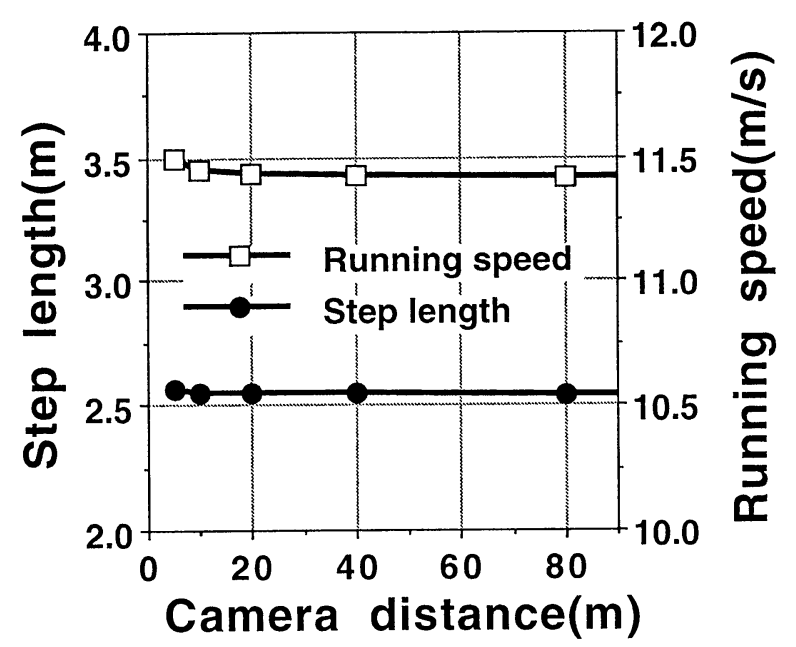

Fig. 4. Change in step length and running speed with the increase in camera distance. The data used to compute step length and running speed is obtained through the filming from 5, 10, 20, 40 and $80 \mathrm{~m}$ of camera distance.

Figure 5 shows the stick figures of a sprinter drawn on the basis of simulated and real two-dimensional coordinates. The camera distances in the simulated data were $5 \mathrm{~m}, 10 \mathrm{~m}$ and $40 \mathrm{~m}$. Relative positions of the limbs in the stick figures based on simulated coordinates, especially for the camera distance of $5 \mathrm{~m}$, were quite different from those based on the real coordinates. The discrepancy was larger near the margins of the filmed area. 


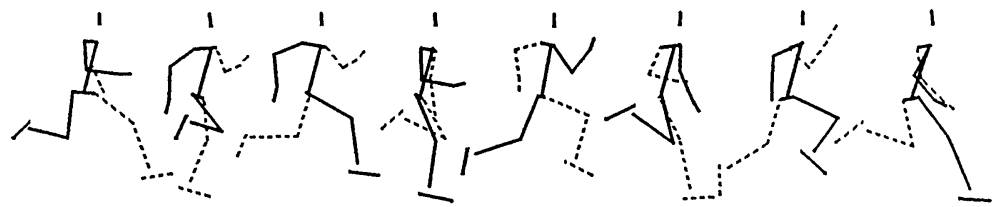

Camera distance $=5 \mathrm{~m}$

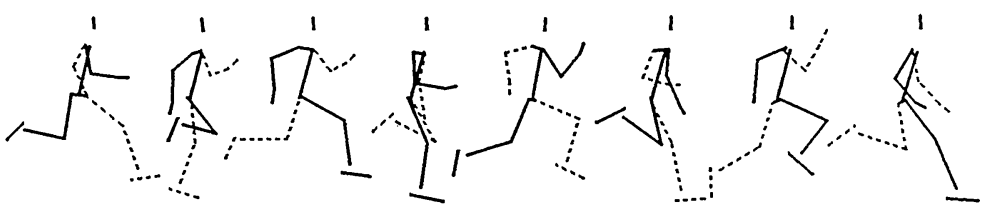

Camera distance $=10 \mathrm{~m}$

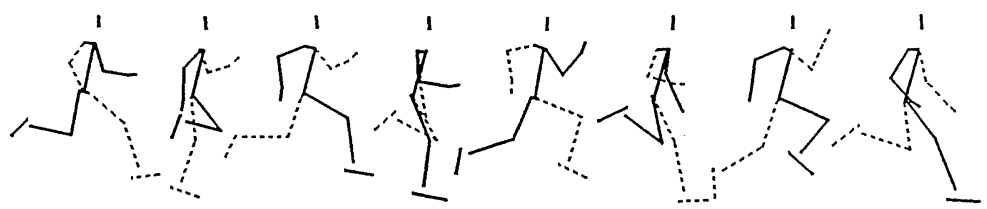

Camera distance $=40 \mathrm{~m}$

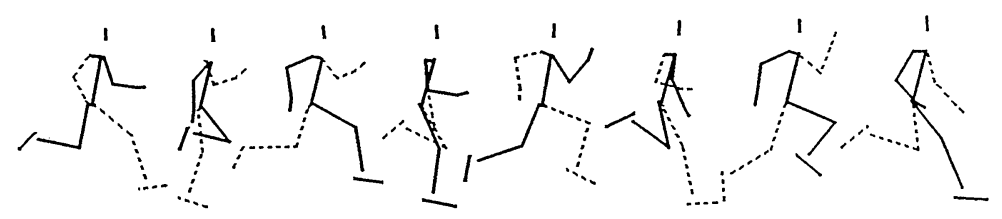

Real coordinates

Fig. 5. Stick figures based on the data through the filming from 5, 20 and $40 \mathrm{~m}$ of camera distance.

The differences recognized in the stick figures may affect the values of joint angles as well as the locations of the segment centers of gravity. Figures 6a and $6 \mathrm{~b}$ show the error of the joint angles for the elbow, hip and knee of the right side. The horizontal axes in these figures show the horizontal location of the whole body center of gravity (WBCG) relative to the origin of the two-dimensional coordinate system. The error of the horizontal locations of the segment centers of gravity for the arm and leg of the right side are shown in Figs. $7 \mathrm{a}$ and $7 \mathrm{~b}$ in the same way as in Figs. $6 \mathrm{a}$ and $6 \mathrm{~b}$. The errors of the joint angles and the locations of the segment centers of gravity become larger with the subject's distance from the origin of the coordinates, i.e., the center of filmed area. The effects of perspective error are larger for the limbs because the limb segments were not parallel to the calibration plane. 
These errors of the joint angles and the locations of the segment centers of gravity may affect the calculated kinetic variables, such as joint torque. However, it is clear that the discrepancies in kinetic variables, which occur due to perspective error, decrease with the increase of camera distance.
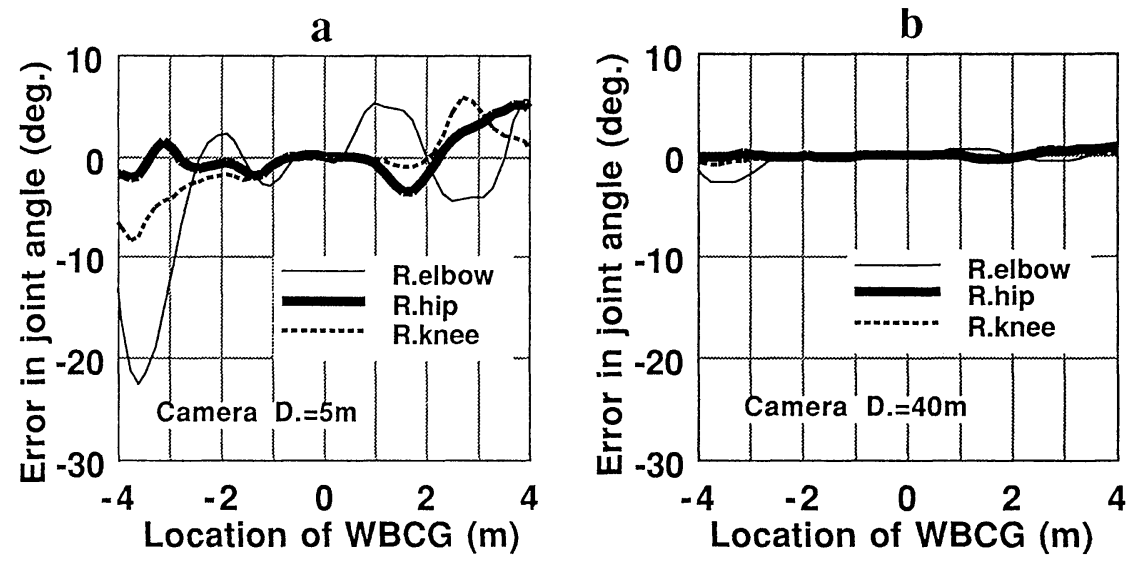

Fig. 6. Change in the error of joint angle with change in the location of the whole body center of gravity (WBCG) relative to the origin of the coordinates system for scale calibration. a: camera distance $=5 \mathrm{~m}$. b: camera distance $=40 \mathrm{~m}$.
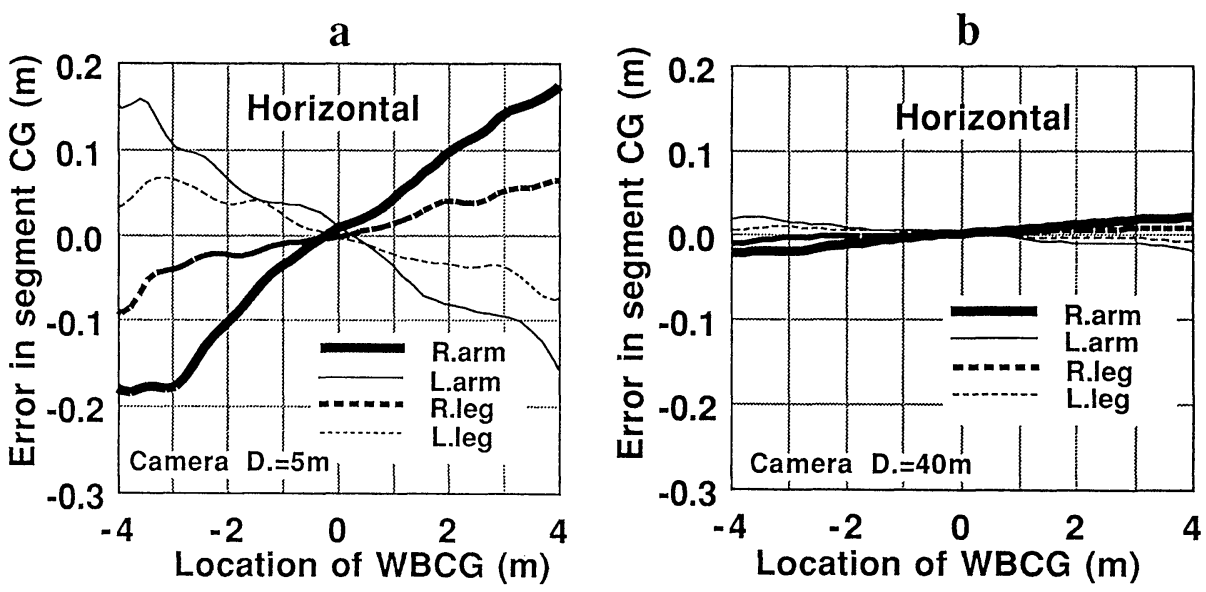

Fig. 7. Change in the error of segment center of gravity with change in the location of the whole body center of gravity (WBCG) relative to the origin of the coordinates system for scale calibration. a: camera distance $=5 \mathrm{~m}$. b: camera distance $=40 \mathrm{~m}$. 
Although the level of acceptable accuracy in kinematic and kinetic variables depends on the purpose of the studies, it seems better to reduce the effects of the perspective error as much as possible. Although the best way for eliminating the perspective error in two-dimensional filming is placing the camera far away from filming objects and/or calibration plane, the camera distance is inevitably limited by the size of the experimental room as mentioned before. Thus data taken at short range need to be corrected. Plagenhoef (1968) considered the correction of the segment length in two-dimensional analysis. His method requires knowledge of both the real segment lengths and the angles between the calibration plane and longitudinal axis of the segment. In practice, however, it is often difficult and complicated to estimate angles and segment lengths from film images.

Since the effects of perspective error are large for the limbs, we tried to correct the data for limb segments. Assuming $L$ to be half of the length between the shoulder joints and/or the hip joints and replacing $d$ in equations (5a) and (5b) by $L$, the equations to obtain real shoulder and/or hip joint locations $(\mathrm{X}, \mathrm{Z})$ are:

$$
\begin{aligned}
& \mathrm{X}=\mathrm{u} \cdot(\mathrm{D} \pm \mathrm{L}) / \mathrm{D} \\
& \mathrm{Z}=\mathrm{v} \cdot(\mathrm{D} \pm \mathrm{L}) / \mathrm{D} .
\end{aligned}
$$

If the locations of landmarks for a limb are assumed to be in a plane parallel to the calibration plane, we might be able to apply equations (5c) and (5d) to estimate the real two-dimensional coordinates of landmarks for the limbs.
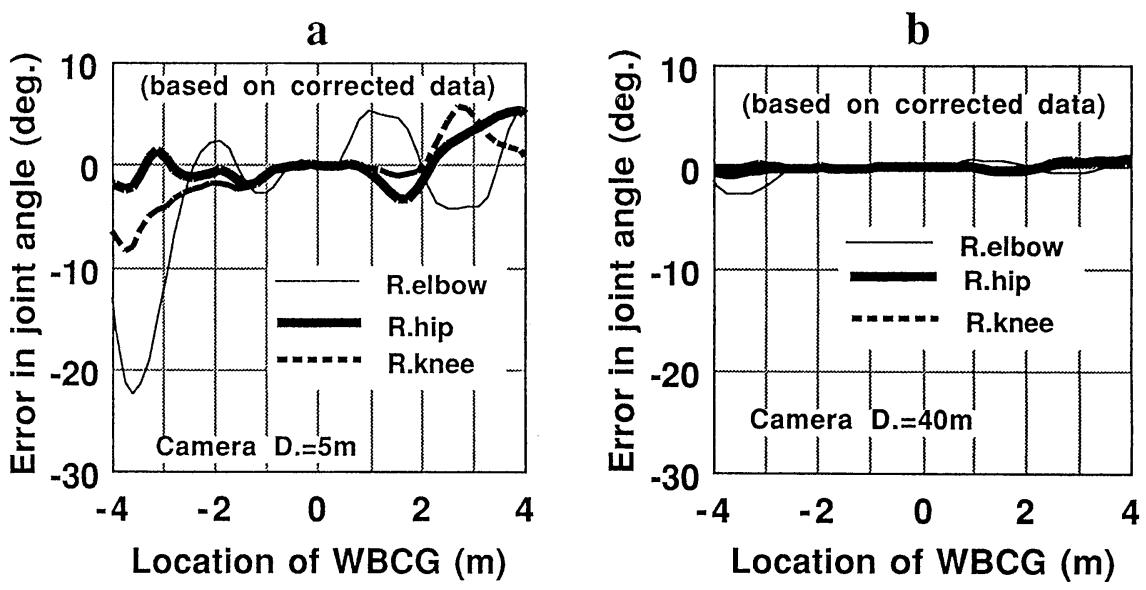

Fig. 8. Change in the error of joint angle with change in the location of the whole body center of gravity (WBCG) relative to the origin of the coordinates system for scale calibration. The coordinates of the landmark locations used for calculating these variables were corrected based on the equations $(5 \mathrm{c})$ and (5d). a: camera distance $=5 \mathrm{~m}$. b: camera distance $=40 \mathrm{~m}$. 
a

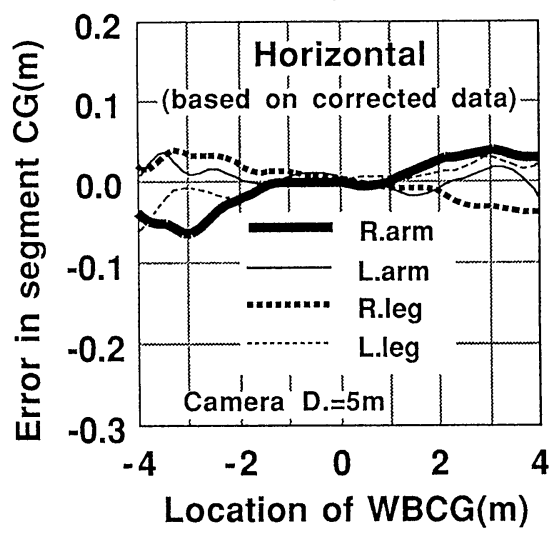

b

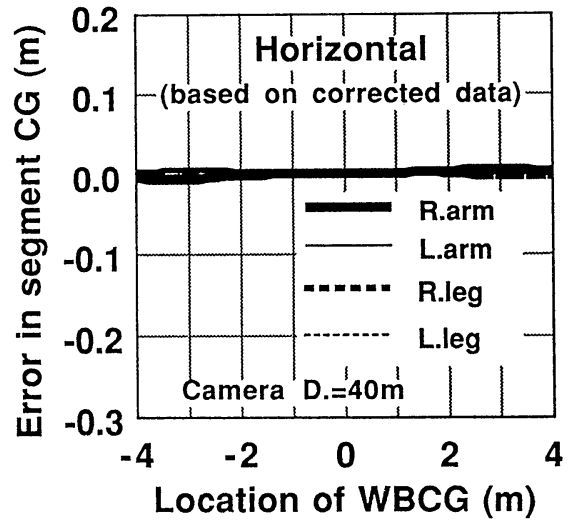

Fig. 9. Change in the error of segment center of gravity with change in the location of the whole body center of gravity (WBCG) relative to the origin of the coordinates system for scale calibration. The coordinates of the landmark locations used for calculating these variables were corrected based on the equations (5c) and (5d). a: camera distance $=5 \mathrm{~m}$. b: camera distance $=40 \mathrm{~m}$.

The simulated two-dimensional coordinates containing a perspective error are corrected according to equations (5c) and (5d). The corrected data from Figs. 6 and 7 are shown in Figs. 8 and 9, respectively. The errors included in the locations of the segment centers of gravity are reduced by the correction, especially for the data with a camera distance of $5 \mathrm{~m}$. The accuracy of joint angles, however, is not improved by the correction because the above correction does not change the relative position of the limb segments, i.e., joint angles, but only changes the sizes of the segments.

It should be noted that application of equations (5a) and (5b) in the correction are not always valid. The data concerning translations of the limb segments seem to be corrected appropriately in the analysis of running as well as walking. However, the effects of the perspective error cannot be eliminated from rotations of limb segments by using equations (5c) and (5d). Therefore, as Noss (1967) suggested, three-dimensional measurements are necessary if the accuracy of segment and/or joint angles is a critical factor in the motion analysis.

\section{SUMMARY}

In the present study, we discussed the perspective errors in two-dimensional motion analysis and proposed equations to estimate the amount of perspective errors using camera distance and object size. Also, we discussed the effects of perspective errors on the kinematic variables in two-dimensional motion analysis of sprint 
running; the perspective error influenced both, translations and rotations of the limb segments which moved outside the calibration plane. We propose the following practical suggestions for two-dimensional motion analysis:

$a$. Equations (4b) and (4c) may be used to estimate the perspective error a priori and for determining the appropriate camera distance.

$b$. In order to reduce the perspective errors, one should select appropriate camera distance based on object's size and the movement depth.

$c$. If the information about the location of optical axis on the calibration plane, which is perpendicular to the axis, is available, effects of perspective error on translational movements of the limb segments can be reduced by using equations $(5 c)$ and $(5 d)$.

\section{ACKNOWLEDGEMENT}

The authors would like to thank Dr. Michiyoshi Ae, associate professor of the University of Tsukuba, for providing us with the three-dimensional data on sprint running.

\section{REFERENCES}

Iwata, H. (1983) A study on the coordination pattern of four limbs in creeping, running and walking in early childhood. J. Anthrop. Soc. Nippon 91(2), 131-152.

Martin, T.P., and Pongrats, M.B. (1974) Mathematical correction for photographic perspective error. Res. Quart. 45(3), 318-323.

Miller, D.I., and Nelson, R.C. (1973) Biomechanics of Sport, Lea \& Febiger, Philadelphia.

Noss, J. (1967) Control of photographic perspective in motion analysis. J. Health, Physical Education and Recreation 38, 81-84.

Plagenhoef, S. (1968) Computer programs for obtaining kinetic data on human movement. $J$. Biomechanics 1, 221-234.

Walton, J.S. (1979) Close-range cine-photogrammetry: Another approach to motion analysis. In Biomechanics Cinematography (Terauds, J., ed.), Academic Publishers, Del Mar, California, pp. 69-97.

Yamada, T., Kondo, S., and Maie, K. (1984) Foot movement during walking with footwear. J. Human Ergol. 13, 137-145. 